\title{
Light scanner based on a viscoelastic stretchable grating
}

\author{
A. N. Simonov, O. Akhzar-Mehr, and G. Vdovin \\ Electronic Instrumentation Laboratory, Delft University of Technology, Mekelweg 4, 2628 CD Delft, The Netherlands \\ Received November 3, 2004 \\ We present a new technique for light scanning by use of viscoelastic-based deformable phase diffraction \\ gratings. Mechanical stretching of the grating permits control of its spatial period, and thus the orders of \\ diffraction can be spatially deflected. In the experiments the viscoelastic gratings with triangular and rect- \\ angular profiles have been characterized at $\lambda=633 \mathrm{~nm}$. It is demonstrated that the reversible elongation can \\ exceed $20 \%$ of the initial length. For the triangular profile grating, the diffraction angle of the first order \\ changed from $6.6^{\circ}$ to $5.4^{\circ}$ while the diffraction efficiency remained almost constant at $\sim 17 \%$. Dynamic scan- \\ ning of a laser beam at frequencies of $\sim 1 \mathrm{kHz}$ is demonstrated by use of electromechanically driven vis- \\ coelastic gratings. (C) 2005 Optical Society of America \\ OCIS codes: $050.1950,050.2770,120.5800,160.2750,230.1360$.
}

Diffraction phase gratings are traditionally used for light deflection and splitting. They have a number of applications in spectroscopy, holography, interferometry, fiber-optic interconnects, light scanners, etc. ${ }^{1,2}$ There has been a growing interest in phase gratings in the past few years brought about by the gratings' essential advantages for ultrafast laser applications. Compared with an ordinary beam splitter, a phase grating permits the generation of passively phaselocked pulse pairs and ensures high phase stability. ${ }^{3,4}$ Another important advantage is that it produces diffraction orders with wave fronts that are not tilted relative to the incident beam. ${ }^{5}$

Optics that employ controllable diffraction elements are of interest for laser beam scanning and deflection. These elements have been used in many applications, including laser treatment of materials, projector systems, and light shutters. Most known devices make use of acousto-optical, electrooptical, magneto-optical, or hybrid spatial light modulators. ${ }^{6-8}$

In this Letter we report on new low-cost transmission diffraction elements with the possibility of controlling their parameters by mechanical deformation. Diffraction gratings with rectangular and triangular profiles are produced by replication from master molds by the use of a two-component viscoelastic silicone elastomer (Sylgard 184, Dow Corning Corporation). We fabricated a master with a rectangular profile by etching an aluminum layer deposited on to a silicone chip. ${ }^{9}$ One-level gray-tone lithography was employed to make the triangular profile master. ${ }^{10,11}$ The newly prepared elastomer is deposited in the master mold and then is cured at $60{ }^{\circ} \mathrm{C}$ for $\sim 8 \mathrm{~h}$ until complete polymerization occurs. The viscoelastic elastomer has low optical losses, perfect homogeneity, and parameter stability. It can be reversibly elongated by $40 \%$ of its initial length. ${ }^{12}$ This property permits the creation of deformable optical elements.

The resultant viscoelastic deformable (VD) gratings perfectly replicate the relief of the master molds. Profile parameters were measured by white-light interferometry. The first sample was a rectangular grating with spatial period $\Lambda \cong 32 \mu \mathrm{m}$ (the duty factor was $10 / 32$ ) and groove height $h \cong 0.62 \mu \mathrm{m}$. The second VD grating had a symmetrical triangular profile with $\Lambda \cong 4.46 \mu \mathrm{m}$ and $h \cong 0.59 \mu \mathrm{m}$. The thickness of both samples in the unstressed state was $d$ $\cong 0.4 \mathrm{~mm}$. The refractive index of the viscoelastic was $n_{0}=1.43 .^{12}$ To facilitate smooth and controllable stretching of the VD samples in experiments we glued the gratings onto the clamps of an optical mount equipped with a digital caliper.

The experimental setup for characterization of the VD gratings is shown in Fig. 1. The light beam of a He-Ne laser at $\lambda \cong 633 \mathrm{~nm}$ coming through the spatial filter (SF) with a $50-\mu \mathrm{m}$ pinhole diffracts off the VD phase grating. The diffraction orders are then collected by an objective $(\mathrm{O})$ with focal length $F=2 \mathrm{~cm}$ onto a CCD camera. The grating is placed in the focal plane of the objective, and the far-field intensity pattern is monitored by the CCD. In the experiments, the intensity distribution for several diffraction orders and the diffraction angle $(\Theta)$ of the 1st order were registered for different values of the VD sample elongation $(l)$.

Figure 2 shows the spatial spectra and intensity cross sections obtained with the rectangular grating in a normal state [Fig. 2(a)] and under stretch [Fig. 2(b)]. The solid curves in Fig. 2 represent theoretically calculated dependencies (teor.) for the measured profile parameters in a normal (unstretched) state. Simulations were performed by use of a standard fast-Fourier-transform-based approach ${ }^{1,13}$ for light diffraction by a thin phase screen. There is good agreement between the experimental and simulated results. Some of the discrepancy is thought to be due

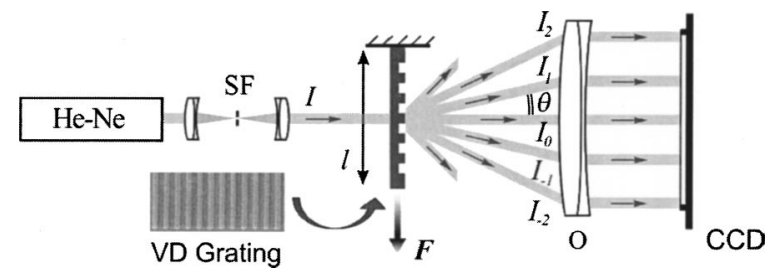

Fig. 1. Experimental setup. 

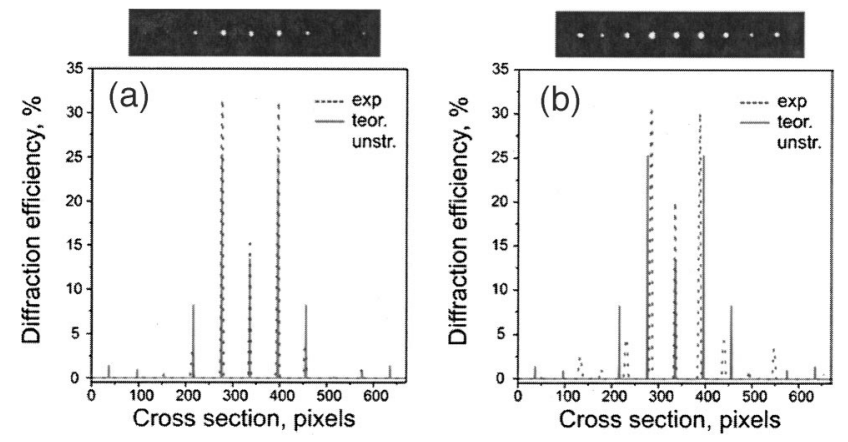

Fig. 2. Spatial spectra of light diffracted by (a) the unstretched $l=18.68 \mathrm{~mm}$ and (b) the stretched $l=22.03 \mathrm{~mm}$ VD grating with a rectangular profile.

to the inaccuracy of the technique that we used to determine the profile parameters.

As can be seen from Fig. 2., the relative elongation $\Delta l / l$ of the grating by $\sim 18 \%$ hardly affects the diffraction efficiency $\left(\eta_{ \pm 1}\right)$ of the \pm 1 orders. Where $\eta_{ \pm 1}$ $=I_{ \pm 1} / I, I$ is the intensity of incident light, and $I_{i}$ is the intensity of the $i$ th diffraction order (see Fig. 1), $i$ $=0, \pm 1, \pm 2$, etc. The 1st-order diffraction angle changes from $\Theta_{a}=1.16^{\circ}$ to $\Theta_{b}=0.94^{\circ}$, whereas the sample length increases from $l_{a}=18.68 \mathrm{~mm}$ to $l_{b}$ $=22.03 \mathrm{~mm}$. This means that the sample thins only slightly and the change in groove height is almost negligible. This result can be explained by the relatively low value of the Poisson ratio of the viscoelastic. Similar results were obtained for the triangular profile grating. In this case the diffraction angle decreased from $\Theta_{a}=6.56^{\circ}$ to $\Theta_{b}=5.36^{\circ}$ at $\Delta l / l \cong 0.18$. Measured efficiency $\eta_{ \pm 1}$ is $\sim 16.5 \%$, whereas the theory predicts $\eta_{ \pm 1} \sim 18.5 \%$. The good correspondence between the actual profile parameters and those specified while the master molds were made opens the way to fabricating deformable optical diffraction elements with desired properties.

The grating profiles used in the experiments were not optimized to attain maximum efficiency in a specific diffraction order. But the computations show that specially designed echelle grating profiles optimized for deflection into a single order combined with an elastic substrate should permit wide scanning angles with high diffraction efficiency (theoretically as much as 100\%). The profile parameters determined in our simulations also satisfy the technological requirements for preparation of the master molds.

Elongation of the VD grating in the longitudinal direction results in its thinning and deformation in the transverse direction. This nonuniform profile deformation leads to optical distortions of the transmitted laser beam. To estimate the magnitude of the distortions, we compared interferometric images of central parts of the gratings (in the 0th order of diffraction) in the normal state and under stretching. A MachZehnder interferometer with a 1:1 sample imaging system was employed in these experiments. Figure 3 presents the interferograms taken for the VD grating with a rectangular profile. As can be clearly seen, the elongation causes aberrations in the wave front of the transmitted beam. The interference patterns have been fitted by the intensity distribution: $I(x, y)$ $\sim C+\cos (2 \pi f / \lambda)$, where $C$ is a constant, $f=f(x, y)$ is the wave-front distortion caused by the grating deformation, and $x$ and $y$ are the coordinates in the sample plane. The astigmatic term was found to be dominating in the Zernike decomposition of $f$. The retention of other aberration terms did not reduce the fit discrepancy significantly. For only an astigmatic fit, i.e., $f=A\left(x^{2}-y^{2}\right)$, we got the amplitude $A \cong 0.75 \lambda$ $(\sim 475 \mathrm{~nm})$. Similarly, for the 1st order of diffraction the dominating astigmatic term was found to be even smaller: $A \cong 0.3 \lambda(\sim 190 \mathrm{~nm})$, but the intensity distribution became more inhomogeneous [see picture (4) in Fig. 3]. The calculated Strehl ratio for a 5-mmdiameter beam in the 1st diffraction order was $\sim 0.53$, whereas for a $1-\mathrm{mm}$ beam it reached $\sim 0.99$. Thus for the majority of applications the deformation-induced aberrations of the light beam diffracted by the VD grating are acceptable.

Dynamic scanning of light can be obtained with the VD gratings driven by the voice coil. Assuming that the sample's elongation leads to a proportional change of its spatial period $(\Delta l \sim \Delta \Lambda)$, for the harmonically driven VD grating we have $\Lambda(t)=\Lambda_{0}[1$ $+\epsilon \sin (\Omega t)]$. Here $\Lambda_{0}$ is the spatial period of the grating in the normal state and $\epsilon$ is the relative amplitude of elongation at frequency $\Omega$. The diffraction angle of the $n$th order, $\Theta_{n}=\arcsin (\lambda n / \Lambda)$, also experiences periodic changes. Expressing the timedependent angle in the form $\Theta_{n}(t)=\Sigma_{p} \Theta_{n}{ }^{(p)} \sin (p \Omega t)$ and stating that $\epsilon \ll 1$, we get for the first-order term $\left(p=1\right.$ in the series): $\Theta_{n}{ }^{(1)} \cong-\lambda n \epsilon / \Lambda_{0}$. So, to achieve larger amplitudes of scanning, one should use the higher orders of diffraction, and $\Lambda_{0}$ has to be maximized along with $\epsilon$.

The typical frequency response of the VD grating is shown in Fig. 4. In the experiment the grating was glued between the clamp of an optical mount and the voice coil. The mechanical response of the voice coil was measured independently to account for its contribution to the resultant response. Resonance peaks
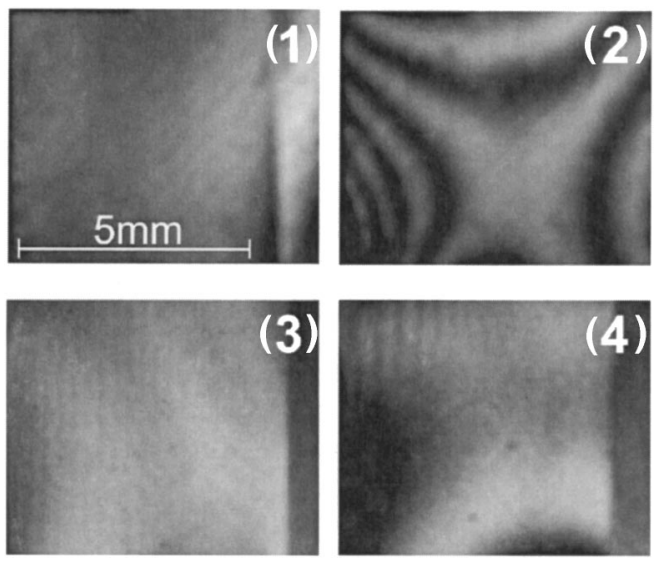

Fig. 3. Interferograms of the VD grating (in the 0th order) in (1) the normal state, $l=18.68 \mathrm{~mm}$ and (2) under stretch, $l=22.03 \mathrm{~mm}$. Similarly, (3) and (4) are interferograms for the 1st order of diffraction. 


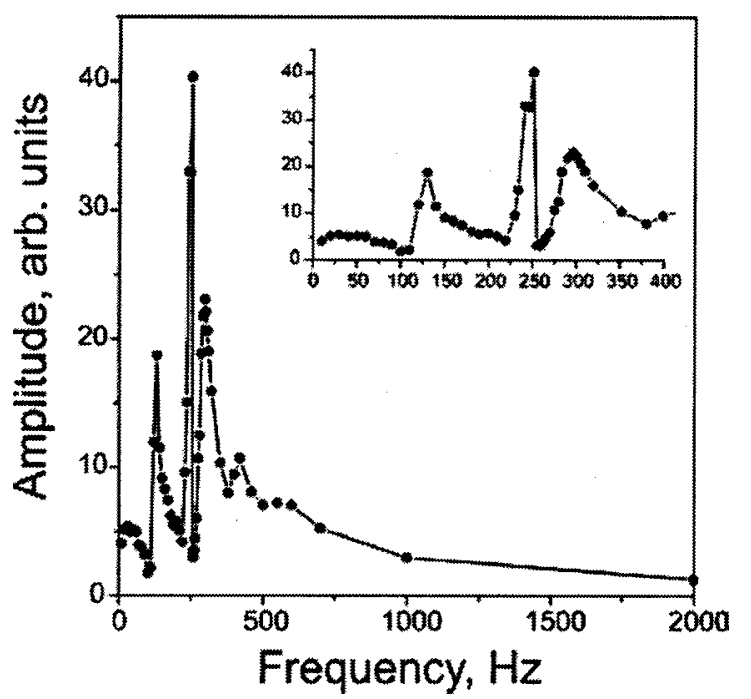

Fig. 4. Frequency response of the electromechanically driven VD grating.

in the plot correspond to the eigenmodes of the VD sample, in the string model: $\omega_{q}=\pi q \sqrt{F / \rho} / l$ $(q=1,2,3 \ldots)$, where $F$ is the grating tension and $\rho$ is its mass density. Note that prestraining and shortening of the VD grating offer a simple way to widen the off-resonance frequency range of the scanner. This widening is of potential interest for integrated micromachined devices.

In conclusion, we have presented a new approach to light scanning by use of viscoelastic-based deformable diffraction elements. This light is deflected by changing the spatial period of a VD grating that had been subjected to mechanical stretch. The VD gratings exhibited excellent stability (determined by the mechanical construction of the VD-based device), low light loss, and a wide range of deflection-as much as $\sim 20 \%$ of the initial angle-and their integratation with mechanical and optical elements was easily accomplished. The optical efficiency and response time of the VD scanner correspond to or are better than those of the micromachined devices for light scanning. ${ }^{14}$ VD gratings are thought to be useful for femtosecond applications, as they facilitate controllable light deflection of wideband light pulses. VD elements can also be used for remote optical sensing of mechanical strain and pressure in a wide range, as was demonstrated by Hosokawa et $a l .{ }^{15}$ for small pressure. The elaborate technology of VD sample fabrication offers a flexible and inexpensive way to make diffraction elements with different parameters for specific applications.

A. N. Simonov's e-mail address is a.n. simonov@ewi.tudelft.nl.

\section{References}

1. M. Born and E. Wolf, Principles of Optics (Cambridge U. Press, Cambridge, 2002), Chaps. 8 and 11.

2. M. Bass, ed., Handbook of Optics (McGraw-Hill, New York, 1995), Vol. I, Chap. 3; Vol. II, Chap. 19.

3. G. D. Goodno and R. J. D. Miller, Chem. Phys. 103, 10619 (1999).

4. A. A. Maznev, K. A. Nelson, and A. Rogers, Opt. Lett. 23, 1319 (1998).

5. A. A. Maznev, T. F. Crimmins, and K. A. Nelson, Opt. Lett. 23, 1378 (1998).

6. G. F. Marshall, ed., Laser Beam Scanning: OptoMechanical Devices, Systems, and Data Storage Optics (Marcel Dekker, New York, 1985).

7. G. F. Marshall, ed., Optical Scanning (Marcel Dekker, New York, 1991).

8. U. Efron, ed., Spatial Light Modulators: Materials, Devices and Systems (Marcel Dekker, New York, 1995).

9. S. Sakarya, "Micromachining techniques for fabrication of integrated light modulating devices," Ph.D. dissertation (Delft University of Technology, Delft, The Netherlands, 2003).

10. W. Henke, W. Hoppe, H. J. Quenzer, P. StaudtFischbach, and B. Wagner, in Proceedings of IEEE Workshop on Micro Electro Mechanical Systems (Institute of Electrical and Electronics Engineers, Piscataway, N.J., 1994), pp. 205-210.

11. K. Reimer, Micro-Optics, Diffractive Elements, Fraunhofer Institute for Silicon Technology http:// www.isit.fhg.de/.

12. Dow Corning Corporation, Product Ref. 10-898E-01 (Dow Corning Corporation, location, Midland, Michigan, 2005); http://www.dowcorning.com/ DataFiles/090007b5802e2039.pdf/.

13. S. Ahmed and E. N. Glytsis, Appl. Opt. 35, 4426 (1996).

14. A. A. Yasseen, S. W. Smith, F. L. Merat, and M. Mehregany, IEEE J. Sel. Top. Quantum Electron. 5, 75 (1999).

15. K. Hosokawa, K. Hanada, and R. Maeda, J. Micromech. Microeng. 12, 1 (2002). 